\title{
PARACOCCIDIOIDOMICOSE DO SISTEMA NERVOSO CENTRAL
}

\author{
RELATO DE CASO
}

\author{
CARLOS EDUARDO AMARAL PEREIRA DA SILVA*, ANDERSON FINOTTI CORDEIRO**, \\ ANGELA MARIA GOLLNER***, SONIA MARIA NEUMANN CUPOLILO****, \\ MARCELO QUESADO-FILGUEIRAS ${ }^{* * * * *}$ MILTON FRANCISCO CURZIO******
}

\begin{abstract}
RESUMO - O envolvimento do sistema nervoso central na paracoccidioidomicose vem ganhando importância dentro do espectro de manifestações clínicas da doença. O primeiro registro na literatura de que o Paracoccidioides brasiliensis seria também capaz de comprometer órgãos do sistema nervoso central foi descrito em 1919 por Pereira \& Jacobs; desde então muitos outros estudos têm demonstrado esta forma de apresentação clínica, sendo que em alguns sua frequência atinge os $27,27 \%$. Descrevemos o caso de uma paciente de 34 anos, residente em Juiz de Fora-MG, admitida com quadro clínico compatível com pneumonia e que durante a internação iniciou sintomatologia cerebelar (náuseas, vômitos, ataxia de marcha e dismetria). A tomografia computadorizada de crânio revelou lesão cerebelar sugestiva de abscesso. O tratamento proposto baseou-se na exerese cirúrgica da lesão e, como a anátomo-patologia mostrasse presença de leveduras em gemulação múltipla ("roda de leme de navio"), diagnóstico de neuroparacoccidioidomicose pôde ser firmado, e o tratamento complementado pela associação de sulfametoxazol-trimetoprim. A paciente evoluiu bem, persistindo discreta paresia e hipoestesia em membro superior direito.
\end{abstract}

PALAVRAS-CHAVE: paracoccidioidomicose, sistema nervoso central, neuroinfecção.

\section{Paracoccidioidomycosis of the central nervous system: case report}

ABSTRACT - The involvement of the central nervous system in paracoccidioidomycosis is more frequent than previously thought. The first reference to the possibility that Paracoccidioides brasiliensis could affect the central nervous system was by Pereira \& Jacobs in 1919. Since then, a great number of other studies has showed this form of clinical behavior and, in some of them, the frequency has ranged $27.27 \%$. We report a clinical case of a 34-year-old white Brazilian woman admitted because of bacterial pneumonia. In the sixth day of admission, the patient developed cerebellar symptomatology with nausea, vomiting, dysmetria and gait disturbance. Central nervous system computer tomographic scanning disclosed a hypodense lesion in the right cerebellar hemisphere. The patient was submitted to surgery with total excision of the lesion. Histopathological examination confirmed the diagnosis of neuroparacoccidioidomycosis. Coadjuvant treatment with sulfamethoxazole-trimetoprim was introduced. The patient had a good outcome and was discharge 30 days after surgery.

KEY WORDS: paracoccidioidomycosis, central nervous system, neuroinfection.

A paracoccidioidomicose é doença sistêmica causada por um fungo dimórfico: o Paracoccidioides brasiliensis. Embora alguns casos isolados tenham sido relatados na América do Norte e Europa , a infecção predomina na América do Sul. No Brasil, onde sua prevalência é maior ${ }^{1}$, observa-se maior incidência nos estados de São Paulo e Minas Gerais ${ }^{2}$. A micose é adquirida através da inalação de conídeos e manifesta-se, principalmente, pelo acometimento mucocutâneo e pulmonar,

Hospital Monte Sinai / Hospital Regional Dr. João Penido - FHEMIG/ Universidade Federal de Juiz de Fora-UFJF: *MD, Neurocirurgião, Professor de Neuroanatomia (UFJF); **MS, Monitor-aluno de Neuroanatomia (UFJF); ***MD, PhD, Professora de Patologia (UFJF); ****MD, MSc, Professora de Patologia (UFJF); *****MD, Neurocirurgião, MSc, Professor de Neurologia (UFJF); ******MD, Neurocirurgião. Aceite: 13-abril-2000.

Dr. Carlos Eduardo A P Silva - Universidade Federal de Juiz de Fora, Instituto de Ciências Biológicas, Departamento de Morfologia, Setor Anatomia - 36036-330 Juiz de Fora MG - Brasil 
frequentemente envolvendo órgãos do sistema retículo-endotelial. O comprometimento do sistema nervoso central (SNC) é mais frequente do que se julgava, atingindo até $27,27 \%$ dos casos em alguns estudos ${ }^{2}$ e podendo acometer a medula, o tronco encefálico, o cerebelo e, mais comumente, os hemisférios cerebrais ${ }^{3}$. Descrevem-se dois tipos clássicos de expressão anátomo-patológica: pseudotumoral e meníngea ${ }^{1-5}$. Algumas séries clínicas têm demonstrado que o envolvimento cerebelar é, também, relativamente frequente, manifestando-se, principalmente, por distúrbios da marcha, tonturas, náuseas, vômitos e diplopia ${ }^{5,6}$.

Relatamos um caso de paracoccidioidomicose disseminada crônica, complicada pelo surgimento de abscesso cerebelar, sendo este tratado, com sucesso, através de drenagem e exerese cirúrgica da cápsula da lesão, complementados com tratamento clínico.

\section{CASO}

LS, 34 anos, feminina, branca, casada, do lar, natural e residente em Juiz de Fora-MG, admitida no Hospital Regional Dr. João Penido em 31 de outubro de 1998 com história de tosse seca, com vários meses de evolução, passando a produtiva nas duas semanas precedentes à internação. Sua expectoração apresentava aspecto purulento, volume moderado e sem hemoptóicos, seguindo-se febre $\left(38,5^{\circ} \mathrm{C}\right)$ persistente e dispnéia aos médios esforços. Emagrecimento de oito quilos em dois meses. Tabagista moderada (5 cigarros/dia durante 20 anos). Ao exame: paciente agudamente doente, estado de nutrição regular, atitude ativa, hipocorada, hipo-hidratada, acianótica, ausência de lesões elementares de pele e/ou mucosas. Sem alterações relativas ao aparelho cardiovascular. O exame do aparelho respiratório revelava taquipnéia ao repouso (32 irpm), ausência de tiragem; à ausculta, observava-se murmúrio vesicular difusamente diminuído, presença de sibilos difusos e crepitações bibasais. Exames complementares na admissão: leucometria 9000/mm³ (segmentado: 79\%, linfócito: 19\%, monócito: $2 \%$, ausência de formas jovens). Radiografia de tórax em PA evidenciou infiltrados e lesões macro e micronodulares em regiões para-hilares bilaterais (Fig 1). Diante de tal quadro levantou-se a hipótese de pneumonia bacteriana comunitária, não descartada a possibilidade de tuberculose pulmonar, sendo iniciado antibioticoterapia empírica com cefalotina $1 \mathrm{~g}$ quatro vezes ao dia. A pesquisa de BAAR no escarro foi negativa em todas as amostras.

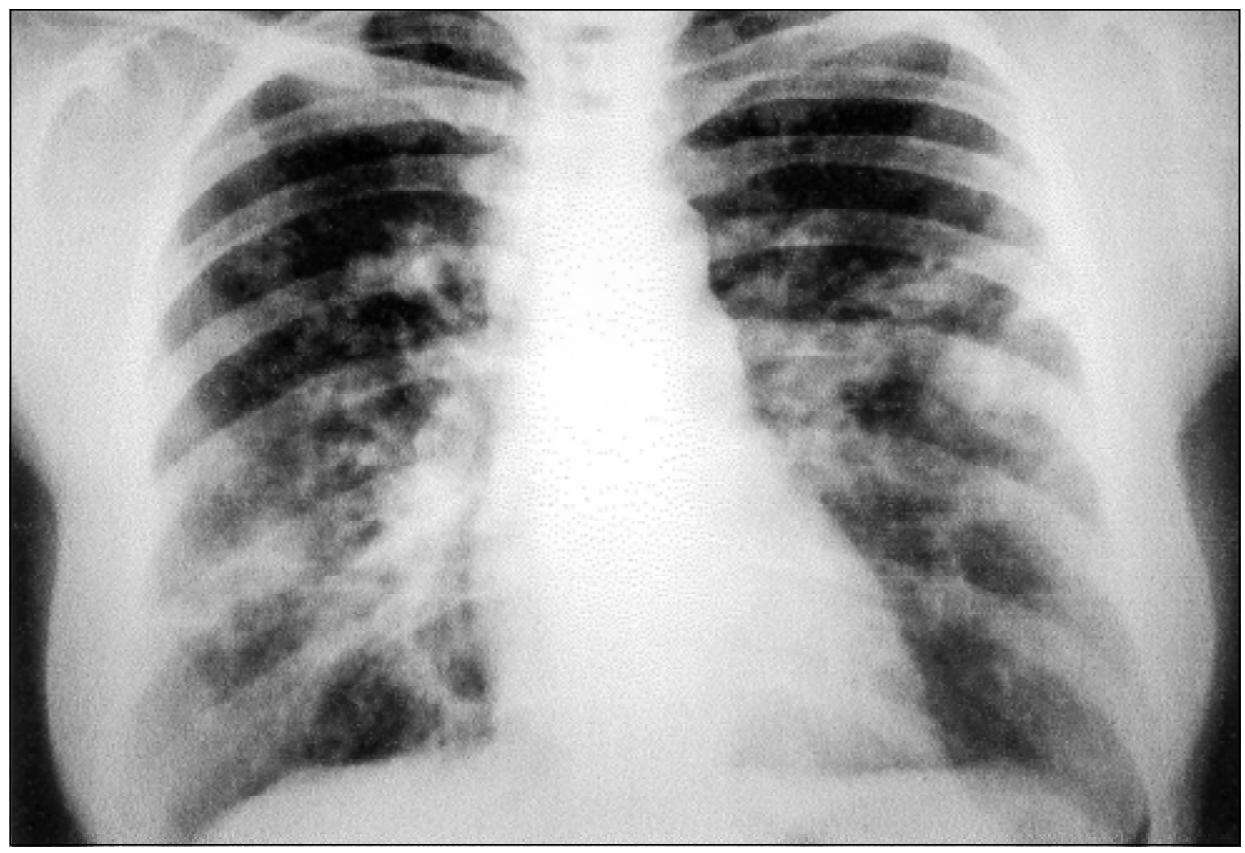

Fig 1. Radiografia de tórax PA obtida na admissão. Observa-se a presença de lesões para-hilares bilaterais típicas do envolvimento pulmonar na paracoccidioidomicose. 


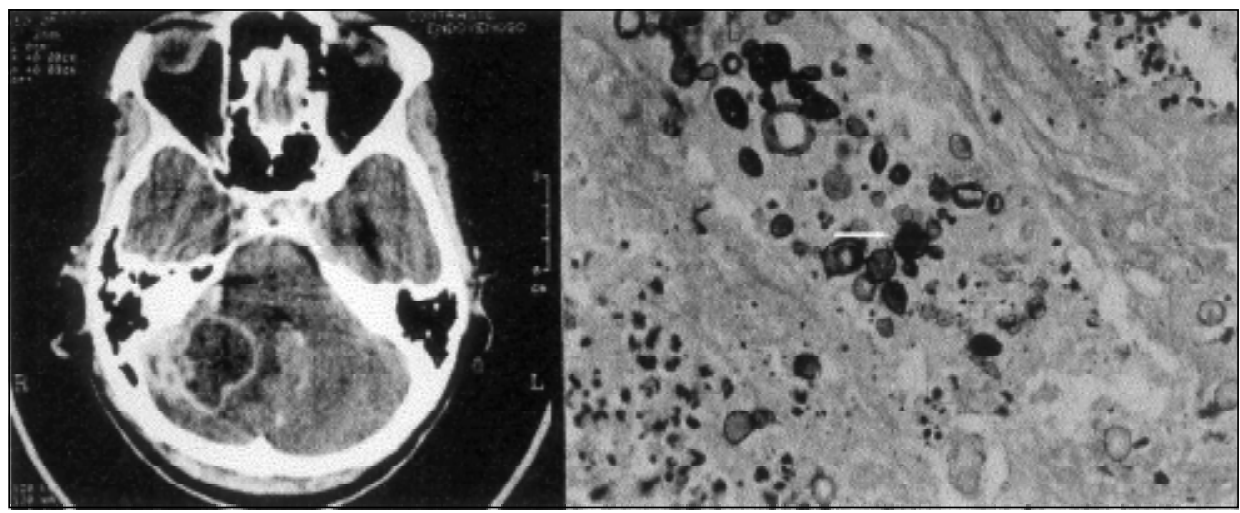

Fig 2A. Tomografia computadorizada de crânio revelando lesão hipodensa com captação anelar de contraste no hemisfério cerebelar direito compatível com abscesso cerebelar. $B$ - Fotomicrografia do material obtido após intervenção cirúrgica e estudo anátomo-patológico, coloração H-E. Presença de leveduras em gemulação múltipla compatíveis com Paracoccidioides brasiliensis $(300 \mathrm{X})$.

No sexto dia de internação, a paciente queixou-se de cefaléia e sensação vertiginosa. Devido à persistência da imagem radiológica, foi procedida broncoscopia. Esta evidenciou brônquios pérvios com saída de secreção francamente purulenta dos brônquios lobares superior e médio esquerdos, sendo coletado broncoaspirado e o material enviado para citologia, baciloscopia e cultura para BK: todos negativos. A paciente evoluiu com piora da cefaléia, persistência da vertigem, náuseas, vômitos e instabilidade de marcha, sendo solicitada avaliação neurológica. O exame neurológico demonstrou prova de Romberg positiva, marcha instável, dismetria no membro superior direito, discreta rigidez de nuca, demais dados sem anormalidades. A tomografia computadorizada (TC) de crânio revelou a presença de lesão única, hipodensa de aproximadamente $4 \mathrm{~cm}$ de diâmetro, com halo hipercaptante em padrão anelar, após injeção de contraste iodado, em hemisfério cerebelar direito, e hidrocefalia não comunicante (Fig 2A).

Optou-se por craniectomia occipital, realizada em 20 de novembro, para excisão cirúrgica da lesão devida aos sinais de compressão do tronco encefálico e obstrução ao fluxo liquórico. Durante o ato cirúrgico, constatouse lesão com cápsula bem delimitada, supurativa, a qual pôde ser completamente ressecada. Os espécimes (pús e cápsula) foram enviados para cultura e teste de sensibilidade a antimicrobianos (TSA) para bactérias, além de estudo anátomo-patológico. Os resultados da cultura e TSA mostraram-se negativos; a anátomo-patologia identificou extensa área de necrose envolta por reação granulomatosa, observando-se grande número de células gigantes multinucleadas de Langerhans fagocitando fungos esféricos, refringentes e em gemulação múltipla, compatíveis com Paracoccidioides sp (Fig 2B). Foram solicitadas sorologias para blastomicose, histoplasmose e paracoccidioidomicose, além da pesquisa de anticorpos anti-HIV pelo método ELISA, sendo apenas positiva para paracoccidioidomicose $(1: 22)$.

Iniciou-se tratamento com sulfametoxazol-trimetoprim na proporção de $2,4 \mathrm{~g}$ de sulfametoxazol e 480 mg de trimetoprim duas vezes ao dia. A paciente recebeu alta hospitalar em 21 de dezembro de 1998 (51 dias após a internação), apresentando melhora clínica significativa tanto do quadro respiratório quanto do ponto de vista neurológico.

No sexto mês após a alta hospitalar procedeu-se controle ambulatorial. A única queixa pertinente era monoparesia do membro superior direito, referindo dificuldades para realizar tarefas domésticas rotineiras como torcer a roupa lavada. Ao exame neurológico: paciente acordada, ativa, orientada, eufásica, sem sinais de meningismo; nervos cranianos: nenhum déficit foi observado; monoparesia do membro superior direito com hiperreflexia, associadas a discreta hipoestesia no mesmo segmento corporal; prova de Romberg negativa, marcha atípica, ausência de dismetria. Exames laboratoriais foram solicitados a fim de melhor avaliar a evolução do quadro infeccioso: hematimetria 4,24 milhões $/ \mathrm{mm}^{3}$; hemoglobina $12,5 \mathrm{mg} / \mathrm{dl}$; hematócrito $38 \%$; leucometria 7500/mm³; TGO 8 mg/dl; TGP 3 UI; LDH 160 UI; glicose 109 mg/dl; sódio $141 \mathrm{mEq} / \mathrm{l}$; potássio 4,4 mEq/l; magnésio $2 \mathrm{mEq} / \mathrm{l}$; cálcio $8,5 \mathrm{mEq} / \mathrm{l}$; fosfatase alcalina $22 \mathrm{mg} / \mathrm{dl}$; proteínas totais $6,0 \mathrm{~g} / \mathrm{dl}$; albumina $3,8 \mathrm{~g} / \mathrm{dl}$; globulinas $2,2 \mathrm{~g} / \mathrm{dl}$, dupla imunodifusão radial para paracoccidioides 1:16. A radiografia de tórax em PA e perfil demonstrou redução das lesões macro e micronodulares, além da remissão do padrão infiltrativo; a TC de crânio revelou completa 


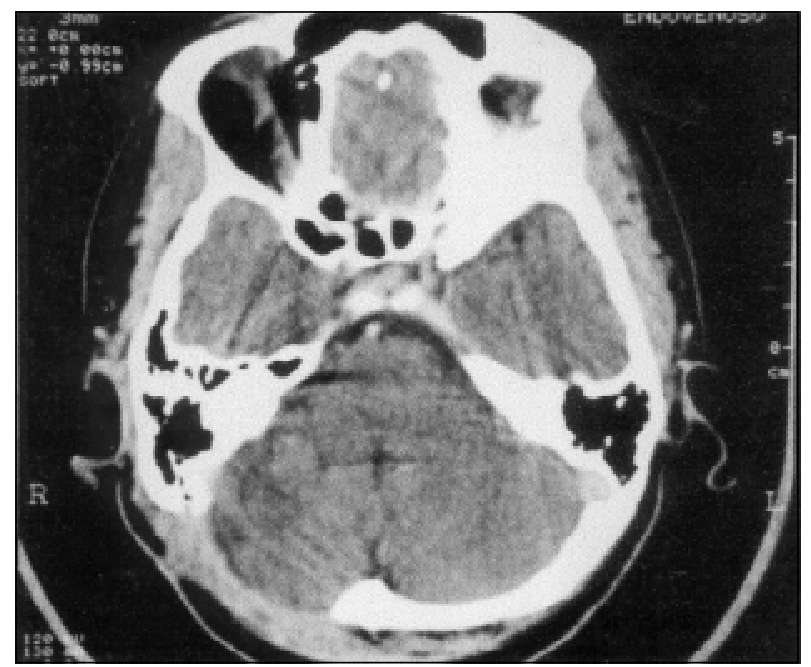

Fig 3. Tomografia copmputadorizada de crânio realizada no sexto mês de pós-operatório. Verifica-se a completa ressecção da lesão, acomodação das estruturas neurais, sendo evidente o local da craniectomia occipital direita.

ressecção da lesão, sem sinais evidentes de recidiva (Fig 3). Foi orientada redução da dose da medicação em uso à metade, devendo esta ser mantida até completar 12 meses, quando nova avaliação será realizada.

\section{DISCUSSÃO}

O Paracoccidioides brasiliensis é um fungo dimórfico, capaz de se desenvolver sob a forma de levedura em meio de cultura a $37^{\circ} \mathrm{C}$ e na forma micelial a temperaturas ambientes que variam entre 4 e $28^{\circ} \mathrm{C}^{7,8}$. As leveduras podem apresentar brotamentos que, quando múltiplos, conferem ao parasita uma conformação semelhante à roda de leme de navio, tendo esta valor diagnóstico ${ }^{7}$. Admitese que o fungo viva saprofiticamente no solo das áreas endêmicas, sendo consideradas como características ecológicas favoráveis ao seu crescimento: 1 . Clima temperado a quente (média de temperatura em torno dos $25^{\circ} \mathrm{C}$ ), pluviosidade anual em torno de 500 a $3500 \mathrm{~mm}^{3}$, com verões chuvosos e invernos curtos e secos; 2. Altitude entre 50 e $1700 \mathrm{~m} ; 3$. Vegetação abundante ${ }^{7-11}, 4$. Algumas culturas agrícolas, particularmente o café 7,11 . Tentativas de se isolar o fungo, diretamente do ambiente ou após inoculação em animais de experimentação, têm se mostrado desanimadoras, confirmando a ainda relativa ignorância acerca do microhabitat do parasita ${ }^{9,11}$.

A doença é endêmica entre os países da América Latina, ocorrendo desde o México $\left(20^{\circ} \mathrm{N}\right)$ até a Argentina ( $\left.35^{\circ} \mathrm{S}\right)$, sendo Brasil, Venezuela, Equador e Peru, os países com maior número de $\operatorname{casos}^{2,7,8,12,13}$. Embora se tenha registro da micose em alguns países da Europa e América do Norte, admite-se que tais pacientes tenham estado previamente em áreas endêmicas. No Brasil, onde a prevalência é maior $\left(5,6\right.$ a 17,5\% em estudos com testes intradérmicos $\left.{ }^{1,12,13}\right)$ a doença incide predominantemente na região Sudeste e em particular nos estados de São Paulo e Minas Gerais ${ }^{2,7}$. A paracoccidioidomicose-doença tem sido constatada em todas as faixas etárias a partir dos três anos de idade ${ }^{7,10}$ preponderando, entretanto, entre os 30 e 50 anos. Descreve-se como paciente típico um indivíduo do gênero masculino, subnutrido, rurícola, que vive ou viveu em íntimo contato com a terra, e sua enfermidade apresenta caráter crônico $^{2,3,8}$. Atualmente, o trato respiratório tem sido considerado como a porta de entrada mais frequente para a infecção, adquirindo-se o patógeno através da inalação dos conídeos. Mais raramente, implantação traumática cutâneo-mucosa poderá ocorrer $^{2,7,14}$, sendo a transmissão inter-humana desconhecida. No espectro de manifestações clínicas 
cita-se em primeiro lugar o envolvimento pulmonar (50 a 100\% dos casos), podendo entretanto atingir qualquer órgão do corpo, sobretudo pele e mucosas, linfonodos, supra-renais, fígado e $\operatorname{ossos}^{1,2,7,8,11,13,14}$. Nestes casos, porém, a via hematogênica constitui a forma de disseminação mais importante do parasita a partir dos pulmões. Alguns estudos têm demonstrado o desenvolvimento de imunodeficiência secundária à paracoccidioidomicose com redução de ambas as respostas humoral e celular. Tal fato pode explicar a ocorrência de infecções oportunistas, como a tuberculose e a criptococose associados à paracoccidioidomicose ${ }^{15}$.

O envolvimento do SNC foi sugerido pela primeira vez em 1919 por Pereira \& Jacobs ${ }^{16}$, mas somente após os anos sessenta é que esta forma de apresentação passou a ser estudada mais minuciosamente ${ }^{1}$. A frequência deste acometimento tem variado muito entre os diversos autores. Assim Plá et al. ${ }^{3}$ demonstraram em séries de autópsias frequência de 0-27,3\%, Colli et al. ${ }^{1}$ encontraram variação de 9,6-12,5\%, Moura et al. ${ }^{2}$ apresentam frequência variando de 9,99-27,27\%, Magalhães et al. ${ }^{6}$ referem comprometimento do SNC em $10 \%$ dos casos, havendo apenas 2 casos relatados na literatura ocorridos em Juiz de Fora-MG ${ }^{14,17}$. Entretanto, lesões neurológicas podem ser menos raras do que se acredita, devido ao fato de frequentemente não haver: 1. Apresentação clínica (formas assintomáticas); 2. Exame neurológico especializado e investigação rotineira do SNC por TC e ressonância nuclear magnética (RNM); 3. Estudo sistematizado atual do SNC em necrópsias ${ }^{2-4}$.

As manifestações neurológicas dependem da localização da(s) lesão(ões), destacando-se os sinais de hipertensão intracraniana (vômitos, cefaléia, papiledema), hemiplegia ou hemiparesia ${ }^{4}$, paralisia de nervos cranianos $^{18}$, convulsões e distúrbios da consciência e humor ${ }^{3,5}$. A possibilidade de envolvimento assintomático é real ${ }^{4}$ e sustentado por estudos de necrópsias que demonstram prevalência de 10 a $36 \%$.

A maioria dos pacientes com comprometimento de SNC apresenta doença disseminada, sendo bastante incomum ser este o único órgão acometido ${ }^{19-21}$. No SNC, o parasita provoca reação granulomatosa no parênquima cerebral ou, menos comumente, nas meninges, sob a forma de meningite crônica predominantemente na base do crânio ${ }^{6}$. Esta última pode decorrer da disseminação do parasita no líquor, após manipulação cirúrgica da lesão, confundindo-se clinicamente com a meningoencefalite tuberculosa ${ }^{4,6}$. A lesão, quando localizada, é circundada por delgada membrana ou cápsula, frequentemente com necrose central contendo numerosos parasitas ${ }^{3,5}$. Uma vez formada, a cápsula não permite livre acesso ao fluxo sanguíneo, reduzindo as defesas naturais e a capacidade de destruir o fungo ${ }^{5}$. Granulomas cerebrais são mais frequentes, podendo acometer também o cerebelo, tronco encefálico e, mais raramente a medula espinhal ${ }^{1-5,7,13,21}$.

De modo geral, a análise de líquido cérebro-espinhal (LCR) mostra-se normal ou pode apresentar pleocitose discreta com predomínio de linfomononucleares ou aumento de proteínas ${ }^{3}$, sendo de baixa sensibilidade e especificidade ${ }^{2}$. Além disso, a pesquisa do parasita, seja por exame direto ou cultura, raramente se mostra positiva ${ }^{2,5,13}$. Métodos imuno-histoquímicos podem se mostrar positivos no líquor, entretanto esta constatação não implica necessariamente lesão do SNC, havendo ainda possibilidade de ocorrerem reações cruzadas, em especial, com a histoplasmose e a criptococose ${ }^{4}$.

Métodos neurorradiológicos de imagem são de fundamental importância na caracterização do envolvimento neurológico na paracoccidioidomicose, especialmente em sua forma pseudotumoral. Estudos com TC usualmente identificam lesões isoladas ou múltiplas, hipodensas, com halo hipercaptante em padrão anelar após injeção de contraste iodado ${ }^{14}$. Há, ainda, pequeno edema perifocal e efeito de massa, a não ser que a lesão esteja localizada no compartimento infratentorial ${ }^{3}$. $\mathrm{O}$ diagnóstico diferencial inclui abscesso cerebral, glioma, tumores metastáticos e neurocisticercose $\mathrm{s}^{3,5,21}$. A análise por RNM supera a sensibilidade da TC na demonstração das lesões de fossa posterior. Entretanto, o elevado custo do método limita sua utilização; além disso, o realce das lesões póscontraste pode permanecer por mais de seis meses após o tratamento ${ }^{6}$.

O tratamento cirúrgico é reservado para os casos em que o granuloma é acessível, a sintomatologia não apresenta melhora com o tratamento clínico e uma rápida solução para o problema 
torna-se necessária ${ }^{1,3,4}$. A presença de lesão com características sugestivas de supuração, sua localização acessível, associados à hidrocefalia e compressão do tronco encefálico, verificados nesta paciente, constituíram-se nas principais indicações cirúrgicas para o caso. O papel dos corticosteróides na proteção do tecido neural e tratamento do edema perilesional permanece controverso ${ }^{1}$. Uma resposta positiva à dexametasona indica ação importante da inflamação e, portanto, do edema no mecanismo de patogênese dos sintomas neurológicos ${ }^{21}$. Contudo, o uso prolongado de corticosteróides em doenças infecciosas para as quais ainda não se estabeleceu terapêutica específica pode determinar disseminação e agravamento das lesões ${ }^{2,21}$. Desta forma, decidimos por não usá-los em razão do aspecto supurativo da lesão e desconhecimento do agente etiológico até identificação anátomo-patológica, optando-se pela descompressão cirúrgica associada ao uso de manitol no per-operatório e cabeceira elevada no pósoperatório como únicas medidas anti-edema, não sendo necessária hiperventilação.

Anfotericina B, derivados azólicos e sulfamídicos compõem o arsenal terapêutico atualmente disponível para o tratamento da paracoccidioidomicose. A escolha da melhor opção terapêutica deverá basear-se não apenas na eficácia e segurança da droga, mas também, e principalmente, no acesso do paciente ao medicamento durante toda a duração do tratamento. Dentre os derivados azólicos, destacam-se o cetoconazol, itraconazol e fluconazol. Destes, apenas o último é capaz de atingir concentrações terapêuticas no LCR, necessitando os demais, doses elevadas para tal fim, frequentemente associadas a efeitos adversos não desprezíveis ${ }^{2,3,7,21,22}$. Apesar de sua potente ação antifúngica, a anfotericina B é extremamente tóxica necessitando ainda, administração intravenosa ${ }^{7}$ e, em razão de sua pequena biodisponibilidade liquórica ${ }^{22}$, pode requerer administração intratecal ${ }^{21}$ na neuroparacoccidioidomicose. Seu uso é reservado aos casos extremamente graves, em que haja risco de vida imediato, ou naqueles com contra-indicações aos outros agentes antifúngicos ${ }^{7,8,22,23}$. No presente estudo, a opção por terapêutica complementar utilizando-se a associação sulfametoxazoltrimetoprim baseou-se em: 1 . Boa eficácia terapêutica ( 70 a $90 \%$ dos casos tratados $)^{7} ; 2$. Observação rara de resistência aos agentes da associação $; 3$. Facilidade de penetração através da barreira hemoliquórica atingindo excelente concentração no líquor ${ }^{7,22}$; 4 . Boa tolerância gastrointestinal; 5 . Custo bastante acessível da medicação; e 6. Distribuição gratuita pela Central de Medicamentos (CEME). Seu uso como monoterapia tem sido relatado em alguns estudos com resultados encorajadores, embora a correta duração da terapia não esteja ainda bem definida ${ }^{3,19,21,23}$.

Mesmo com as medidas terapêuticas adequadas, a mortalidade pode superar os $20 \%$ nas formas juvenis e disseminadas ${ }^{3}$. Assim, o diagnóstico precoce da neuroparacoccidioidomicose é particularmente importante, uma vez que há evidências de que uma terapêutica eficiente instituída em tempo hábil é crucial para reduzir os efeitos devastadores da doença e poder levar a cura.

\section{Conclusão}

O acometimento do SNC na paracoccidioidomicose é mais frequente do que se supunha até recentemente. Seu diagnóstico é particularmente difícil sendo que muitos casos são desvendados somente após cirurgia e estudo anátomo-patológico, devendo ser incluído no diagnóstico diferencial das meningoencefalites e tumores do SNC, especialmente nos pacientes provenientes de áreas endêmicas e com evidências clínicas de infecção. O tratamento cirúrgico, combinado à terapêutica antimicrobiana eficaz, deverá ser sempre considerado em lesões localizadas e expansivas do SNC. Sulfametoxazol-trimetoprim mostra-se eficiente como monoterapia e seu uso pode ser justificado por uma boa eficácia terapêutica e baixo custo de tratamento. Por fim, uma evolução favorável está intimamente correlacionada à precocidade diagnóstica e a uma correta decisão terapêutica.

Agradecimentos - Somos gratos à Professora Maria Cristina Vasconcellos Furtado por suas valiosas sugestões e ao Professor Gláucio Mendes Franco pela revisão do manuscrito.

\section{REFERÊNCIAS}

1. Colli BO, Assirati JR JA, Machado HR, et al. Intramedullary spinal cord paracoccidioidomycosis. Arq Neuropsiquiatr 1996;54:466-473.

2. Moura LP, Raffin CN, Del Negro GMB, Ferreira MS. Paracoccidioidomicose evidenciando comprometimento medular tratada com sucesso por fluconazol. Arq Neuropsiquiatr 1994;52:82-86. 
3. Plá M del P, Hartung C, Mendoza P, Stukanoff A, Moreno MJ. Neuroparacoccidioidomycosis: case reports and review. Mycopathologia 1994;127:139-144.

4. Nóbrega JPS, Mattosinho-França LC, Spina-França A. Neuroparacoccidioidomicose. In Del Negro G, Lacaz CS, Fiorillo AM. Paracoccidioidomicose: blastomicose sul-americana. São Paulo: Sarvier - Ed. da Universidade de São Paulo, 1982: 221-227.

5. Rodacki MA, Toni Gde, Borba LA, Oliveira GG. Paracoccidioidomycosis of the central nervous system: CT findings. Neuroradiology 1995; 37: 636-641.

6. Magalhães ACA, Bacheschi LA, Caramelli P, et al. Paracoccidioidomicose do sistema nervoso central: estudo de cinco casos por ressonância nuclear magnética. Rev Hosp Clin Fac Med S Paulo 1993;48:94-97.

7. Veronesi R, Focaccia R. Veronesi: tratado de infectologia. 9Ed. São Paulo: Atheneu, 1997: 1081-1111.

8. Wanke B. Micoses Profundas. In Schechter M, Marangoni DV. Doenças infecciosas: conduta diagnóstica e terapêutica. 2Ed. Rio de Janeiro: Guanabara Koogan, 1998: 318-323.

9. Mcewen JG, Garcia AM, Ortiz BL, Botero S, Restrepo A. In search of the natural habitat of paracoccidioides brasiliensis. Arch Med Res 1995;26:305-306.

10. Rios Gonçalves AJ, Londero AT, Terra GMF, Rozembaum R, Abreu TF, Nogueira SA. Paracoccidioidomycosis in children in the state of Rio de Janeiro (Brazil): geographic distribution and study of a "reservarea". Rev Inst Med Trop S Paulo 1998;40:11-13.

11. Silva-Vergara ML, Martinez R, Chadu A, Madeira M, Freitas-Silva G, Maffei CML. Isolation of a Paracoccidioides brasiliensis strain from the soil of a coffee plantation in Ibiá, State of Minas Gerais, Brazil. Med Mycol 1998;36:37-42.

12. Fava SC, Fava C Neto. Epidemiologic surveys of histoplasmin and paracoccidioidin sensitivity in Brazil. Rev Inst Med Trop S. Paulo 1998;40:155-164.

13. Morato-Fernandez RN, Beraldo PSS, Masini M, Costa PHC. Paracoccidioidomicose de localização intramedular e cerebral. Arq Neuropsiquiatr 1991;49:192-197.

14. Franco GM, Hallack KA, Souza SF, Stroppa ALPC, Hadad DJ. Granuloma de cerebelo: relato de um caso. HU Rev $1985 ; 12: 51-58$

15. Shikanai-Yasuda MA, Segurado AAC, Pinto WP, et al. Immunodeficiency secundary to juvenile paracoccidioidomycosis: associated infections. Mycopathologia 1992;120:23-28.

16. Pereira JM, Jacobs F. Um caso de blastomicose cutânea com acessos epilépticos. An Paul Med Cirurg 1919;10:217-219.

17. Brandão CBPA, Baldi J, Stroppa AT et al. Blastomicose sul-americana: abscesso cerebral e forma pulmonar tumoral. HU Rev 1974:137-141.

18. Dantas AM, Yamane R, Camara AG. South american blastomycosis: ophthalmic and oculomotor nerve lesions. Am J Trop Med Hyg 1990;43:386-388.

19. Argollo A, Reis VLL, Niemeyer P, Vuono E. Central nervous system involvement in South American blastomycosis. Trans R Soc Trop Med Hyg 1978;72:37-39.

20. Salaki JS, Louria DB, Chmel H. Fungal and yeast infections of the central nervous system. Medicine 1984;63:108-132.

21. Valle ACF, Skacel M, Costa RLB, Ribeiro CT, Montagna NAA, Cruz LCH. A case report of intraspinal paracoccidioidomycosis. Rev Inst Med Trop S. Paulo 1998;40:203-207.

22. Mandel GL, Petri WA Jr. Fármacos antimicrobianos (continuação): sulfonamidas, trimetoprima-sulfametoxazol,quinolonas e agentes para infecções das vias urinárias. In Gilman AG, Rall TW, Nies S, Taylor P. Goodman \& Gilman: as bases farmacológicas da terapêutica. 9Ed. Rio de Janeiro: Guanabara Koogan, 1996:777-789.

23. Guerreiro CAM, Chuiluc SSD, Branchini MLN. A new treatment for large cerebral paracoccidioidomycosis. Arq Neuropsiquiatr 1987;45:419-423. 